\title{
Hepatology Consultants Often Disagree on Etiology of Abnormal Liver Biochemistries in COVID-19 but Agree on Management
}

\author{
Patricia P. Bloom ${ }^{1,2}(1) \cdot$ Trisha S. Pasricha ${ }^{1,2} \cdot$ Karin L. Andersson ${ }^{1,2} \cdot$ Daniel S. Pratt $^{1,2} \cdot$ Nikroo Hashemi $^{2,3}$. \\ Irun Bhan ${ }^{1,2} \cdot$ Kathleen Viveiros ${ }^{2,3}$
}

Received: 13 May 2020 / Accepted: 11 July 2020 / Published online: 29 July 2020

(c) Springer Science+Business Media, LLC, part of Springer Nature 2020

\begin{abstract}
Background Coronavirus disease 2019 (COVID-19) is associated with elevated liver biochemistries in approximately half of hospitalized patients, with many possible etiologies.

Aim To assess agreement on the etiology of abnormal liver biochemistries and diagnostic recommendations in COVID-19. Methods Twenty hepatology consultations were reviewed by three senior hepatologists who provided a differential diagnosis and diagnostic recommendations. Kappa agreement on the primary etiology was calculated.

Results Kappa agreement between hepatologists on the primary etiology of elevated liver biochemistries was $0.10(p=0.03)$. Agreement was greater around drug-induced liver injury $0.51(p<0.0001)$ and SARS-CoV-2-related liver injury 0.17 $(p=0.03)$. Serial liver biochemistries were recommended in all consultations over other evaluations.

Conclusion In COVID-19, elevated liver biochemistries present a diagnostic challenge and can often be monitored conservatively.
\end{abstract}

Keywords COVID-19 $\cdot$ Hepatology consultation $\cdot$ Liver biochemistries $\cdot$ Diagnosis

\section{Introduction}

Coronavirus disease 2019 (COVID-19) is associated with elevated liver biochemistries in approximately half of hospitalized patients [1-4]. COVID-19 has been most frequently associated with an aspartate aminotransferase (AST)-predominant hepatocellular injury pattern, though other patterns exist [4-6]. Multiple potential etiologies for elevated liver biochemistries in COVID-19 have been suggested, including direct SARS-CoV-2 liver injury, drug-induced liver injury (DILI), non-liver origin, and ischemia, as well as etiologies not specific to COVID-19 such as cholestasis of sepsis and chronic liver disease [5, 7, 8]. In this context,

Patricia P. Bloom

ppbloom@med.umich.edu

1 Division of Gastroenterology, Department of Medicine, Massachusetts General Hospital, 15 Parkman St, Wang 5, Boston, MA 02140, USA

2 Department of Medicine, Harvard Medical School, Boston, MA, USA

3 Division of Gastroenterology, Department of Medicine, Brigham Women's Hospital, Boston, MA, USA hepatologists are consulted for diagnostic and therapeutic guidance [9]. Our aim was to assess degree of hepatologist agreement on the etiology of abnormal liver biochemistries as well as diagnostic recommendations in COVID-19.

\section{Methods}

We reviewed the characteristics of twenty inpatient hepatology consultations performed at Massachusetts General Hospital and Brigham and Women's Hospital for abnormal liver biochemistries in adult patients with a positive SARSCoV-2 RT-PCR. Three senior hepatologists independently reviewed the data available at the time of consultation. They provided a rank-order list of up to three possible etiologies and any additional testing they would request. Reviewers provided post-discharge recommendations after receiving the liver biochemistries at discharge. A Fleiss kappa statistic was used to calculate agreement between multiple reviewers, using SAS version 9.4 (Cary, NC). This study was deemed exempt by our institutional review board. 


\section{Results}

From March 20 to April 21, 2020, our inpatient hepatology teams performed 20 consultations on patients with COVID19 and abnormal liver biochemistries; eight had chronic liver disease, and twelve required ICU admission (Table 1 for patient characteristics).

Seventeen (85\%) patients had at least one abnormal liver biochemistry on admission. Mean (range) AST at consultation

Table 1 Patient characteristics

\begin{tabular}{ll}
\hline Characteristic & $\begin{array}{l}\text { Entire } \\
\text { cohort } \\
(N=20)\end{array}$ \\
\hline Age, years & $46 \pm 14$ \\
Male, $N(\%)$ & $18(90 \%)$ \\
Hispanic, $N(\%)$ & $11(55 \%)$ \\
Diabetes, $N(\%)$ & $1(5 \%)$ \\
Body mass index & $32 \pm 6$ \\
Pregnant or peri-partum, $N(\%)$ & $2(10 \%)$ \\
Chronic liver disease, $N(\%)$ & $8(40 \%)$ \\
Alcohol-related cirrhosis & $2(10 \%)$ \\
Post-liver transplant & $1(5 \%)$ \\
Nonalcoholic fatty liver disease (NAFLD) & $4(20 \%)$ \\
NAFLD and chronic hepatitis C virus infection & $1(5 \%)$ \\
\hline
\end{tabular}

Data are presented as median \pm SD unless otherwise specified was 343 U/L (41-3300), ALT 288 U/L (25-1591), alkaline phosphatase $223 \mathrm{U} / \mathrm{L}$ (51-976), and total bilirubin $4.0 \mathrm{mg} / \mathrm{dL}$ (0.3-19.3). At the time of hepatology consultation, $10(50 \%)$ patients had a hepatocellular pattern of liver injury, 7 (35\%) had a cholestatic pattern, and $3(15 \%)$ had a mixed pattern, determined by the ALT to alkaline phosphatase ratio [10].

The suspected diagnosis by the original consultant and three senior hepatologists is shown in Fig. 1. The original consultant and three hepatology reviewers had Kappa agreement of $0.10(p=0.03)$ for the most likely primary etiology of elevated liver biochemistries. The two most common diagnoses were SARS-CoV-2-related liver injury and DILI. Between all four hepatologists, there was 0.17 Kappa agreement $(p=0.03)$ that SARS-CoV-2-related liver injury was on the differential and 0.51 Kappa agreement $(\mathrm{p}<0.0001)$ that DILI was on the differential.

Of the 14 patients with at least one reviewer suspecting DILI, 9 (64\%) had hepatocellular, 4 (29\%) had cholestatic, and $1(7 \%)$ had mixed liver injury patterns. The medications administered to those patients included: hydroxychloroquine in 13 (93\%), cephalosporin 13 (93\%), acetaminophen 13 (93\%), statin 12 (86\%), azithromycin 11 (79\%), placebocontrolled remdesivir trial $5(36 \%)$, lopinavir-ritonavir 1 (7\%), and tocilizumab 1 (7\%).

Prior to consultation, medical teams performed the following evaluation: hepatitis B virus (HBV) serologies (85\%), HCV antibody (80\%), HCV PCR (15\%), hepatitis A virus (HAV) serologies (15\%), ultrasound (15\%), ultrasound with Doppler (30\%), and cross-sectional abdominal imaging (5\%).
Fig. 1 Etiology of elevated liver biochemistries in COVID-19. a Number of cases with each primary diagnosis by reviewer. b Global frequency of each diagnosis in the top 3 differential. Includes diagnoses made by original consultant and 3 senior hepatologists

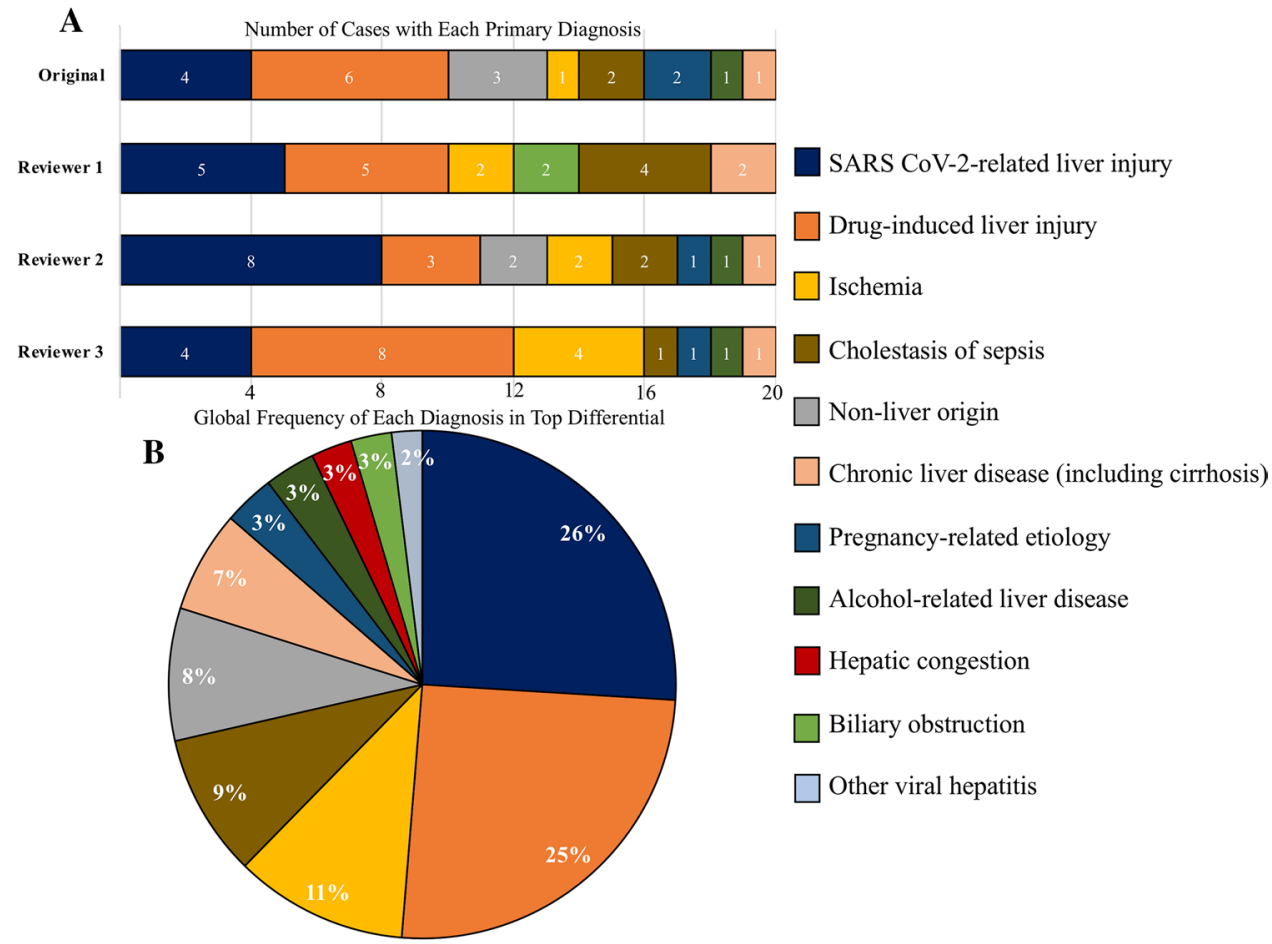


The hepatology consultant recommended: liver biochemistry monitoring (100\%), HBV PCR (10\%), HAV serologies (10\%), ultrasound (5\%), ultrasound with Doppler (15\%), autoimmune markers $(15 \%)$, and other viral studies $(20 \%)$. They did not recommend liver biopsy or cross-sectional imaging in any initial consultation. They recommended against ultrasound in $40 \%$ for lack of necessity and to minimize health care worker exposure. Hepatologist reviewers made similar diagnostic recommendations.

Seven patients were discharged, six had improved liver biochemistries, and none died at the end of the study period on April 22, 2020. For the 7 discharged patients, hepatologist reviewers recommended hepatology follow-up in 3 $(43 \%)$ and liver biochemistries in $6(86 \%)$.

\section{Discussion}

Determining the cause of elevated liver biochemistries in patients with COVID-19 is a challenge. The patients described here were a heterogeneous group by liver disease history and degree and pattern of liver biochemistry elevation. The differential diagnosis includes SARS-CoV2-related liver injury, DILI, complications of the infection including myositis (particularly when AST > ALT), ischemia, and more. The challenge is highlighted by the fact that, without liver biopsy, there was little agreement between hepatologists regarding the most likely etiology of elevated liver biochemistries.

The hepatologists attributed approximately half of cases to phenomena specific to the COVID-19 pandemic: SARSCoV-2-related liver injury or DILI. The remaining cases were attributed to non-COVID-specific etiologies. The greatest consensus was around DILI diagnosis. Patients with suspected DILI were on multiple potentially hepatotoxic drugs, which underscores the complexity of these consults, as well as the challenge of assessing hepatic safety of COVID-19-specific therapies.

These consultations were performed in March and April 2020, early in the US pandemic, at large academic institutions, in a city with relatively high incidence of disease, and mainly for patients with severe liver biochemistry elevations. We suspect that issues related to hepatology consultation will differ by phase of the pandemic, patient population, location, and as more literature about SARS-CoV-2 and its treatments become available. Our data are also limited by lack of a gold standard comparison (i.e., liver biopsy) or extended follow-up.

Despite lack of consensus about etiology of liver biochemistries in inpatient hepatology consultations, there was agreement that the diagnostic strategy for the majority of cases should be serial liver biochemistry monitoring, as opposed to multiple diagnostic tests, especially those that expose health care workers to SARS-CoV-2. Nevertheless, liver biopsy should be considered in select cases of severe liver injury with multiple potential causes. These comments are consistent with expert guidance, which suggests limiting transportation of patients with COVID-19 for liver-related testing unless it is likely to change management [9].

Author's contribution PPB contributed to study concept and design, acquisition of data, data analysis, drafting manuscript, statistical analysis; TSP, KLA, DSP, NH contributed to acquisition of data, critical revision of manuscript; IB contributed to critical revision of manuscript; KV contributed to study concept and design, critical revision of manuscript, study supervision.

\section{Compliance with Ethical Standards}

Conflict of interest None of the authors have conflict of interest to disclose.

\section{References}

1. Huang C, Wang Y, Li X, et al. Clinical features of patients infected with 2019 novel coronavirus in wuhan, china. Lancet. 2020;395:497-506. https://doi.org/10.1016/S0140-6736(20)30183 -5 .

2. Cai Q, Huang D, Yu H, et al. Characteristics of liver tests in COVID-19 patients. J Hepatol. 2020. https://doi.org/10.1016/j. jhep.2020.04.006.

3. Fan Z, Chen L, Li J, et al. Clinical features of COVID-19-related liver functional abnormality. J Clin Gastroenterol Hepatol. 2020;18:1561-1566. https://doi.org/10.1016/j.cgh.2020.04.002.

4. Richardson S, Hirsch JS, Narasimhan M, et al. Presenting characteristics, comorbidities, and outcomes among 5700 patients hospitalized with COVID-19 in the new york city area. JAMA. 2020. https://doi.org/10.1001/jama.2020.6775.

5. Bloom PP, Meyerowitz EA, Reinus Z, et al. Liver biochemistries in hospitalized patients with COVID-19. Hepatology. 2020. https ://doi.org/10.1002/hep.31326.

6. Schaefer EAK, Arvind A, Bloom PP, Chung RT. Interrelationship between coronavirus infection and liver disease. Clin Liver Dis (Hoboken). 2020;15:175-180. https://doi.org/10.1002/cld.967.

7. Jothimani D, Venugopal R, Abedin MF, Kaliamoorthy I, Rela M. COVID-19 and liver. J Hepatol. 2020. https://doi.org/10.1016/j. jhep.2020.06.006.

8. Piano S, Dalbeni A, Vettore E, et al. Abnormal liver function tests predict transfer to intensive care unit and death in COVID-19. Liver Int. 2020. https://doi.org/10.1111/liv.14565.

9. Fix OK, Hameed B, Fontana RJ, et al. Clinical best practice advice for hepatology and liver transplant providers during the COVID19 pandemic: AASLD expert panel consensus statement. Hepatology. 2020. https://doi.org/10.1002/hep.31281.

10. Kwo PY, Cohen SM, Lim JK. ACG clinical guideline: evaluation of abnormal liver chemistries. Am J Gastroenterol. 2017;112:1835. https://doi.org/10.1038/ajg.2016.517.

Publisher's Note Springer Nature remains neutral with regard to jurisdictional claims in published maps and institutional affiliations. 